\title{
A six-year-old boy with autism and left hip pain
}

\author{
Stephanie Erdle MD, Melanie Conway MD, Michael Weinstein MD
}

Cite as: CMAJ 2017 February 21;189:E275-8. doi: 10.1503/cmaj.160712

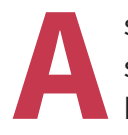

six-year-old boy with autism spectrum disorder presented to the emergency department with a one-week history of left hip pain and limping. His associated symptoms included fever, rhinorrhea and cough. Bilateral hip and knee radiographs were normal, and he was discharged home with a diagnosis of transient synovitis.

The patient's fever and infectious symptoms resolved, but the pain in his left hip worsened. He returned to the emergency department seven weeks later after he had been unable to bear weight for one week. There were no constitutional symptoms (e.g., fever, night sweats or weight loss). He did not have other joint involvement. He had one soft bowel movement daily, with no blood or mucus, and had no abdominal pain. There were no notable findings on review of systems other than the parents noting that he had "red dots" on his arms and legs. He was not on any medications, and was fully immunized.

Repeat radiographs of the patient's hips showed periarticular osteopenia bilaterally, most marked in the right hip (Figure 1).

\section{What diagnoses should be ruled out in this patient?}
a. Slipped capital femoral epiphysis
b. Septic arthritis
c. Osteomyelitis
d. Malignant disease
e. All of the above

The answer is (e). Multiple conditions may cause hip pain in children, but it is essential to rule out serious conditions, such as those listed above. The radiograph did not show evidence of slipped capital femoral epiphysis, which typically occurs in older children. Legg-Calve-Perthes disease is also an important cause of hip pain, but the presentation is usually more insidious. There was also no avascular necrosis seen on the radiographs, although initial radiographs can appear normal.

Septic arthritis should always be considered in a patient with joint pain, although it is typically more acute in onset and less likely without fever. Osteomyelitis is also less probable in a patient who is afebrile. Malignant disease should be considered in any case of bony pain; about $25 \%$ of children with newly diagnosed leukemia present with a complaint of severe bone pain. The patient had no constitutional symptoms, but the parents described "red dots," which could be indicative of thrombocytopenia in the setting of leukemia.

On physical examination, the patient's weight was $21.2 \mathrm{~kg}$ (25th percentile) and his height was $121 \mathrm{~cm}$ (50th percentile). He was afebrile, and vital signs were within normal limits. He appeared pale and sat with his left hip flexed and externally rotated, with flexion of the left knee. He was unable to fully extend his left knee and had discomfort on internal rotation of his left hip. There was no evidence of erythema or warmth, and he had no discomfort on palpation. The range of motion of his right hip and knee were normal, and there were no joint effusions. Hepatosplenomegaly and lymphadenopathy were absent. Dermatologic examination showed palpable petechiae on his arms and legs bilaterally (Figure 2) and some ecchymoses on his legs. The patient had swollen, purple gingiva with blood-filled blisters (Figure 3), which bled spontaneously on examination. His parents reported that these lesions had begun about three months earlier.

Results of initial laboratory tests are shown in Box 1.

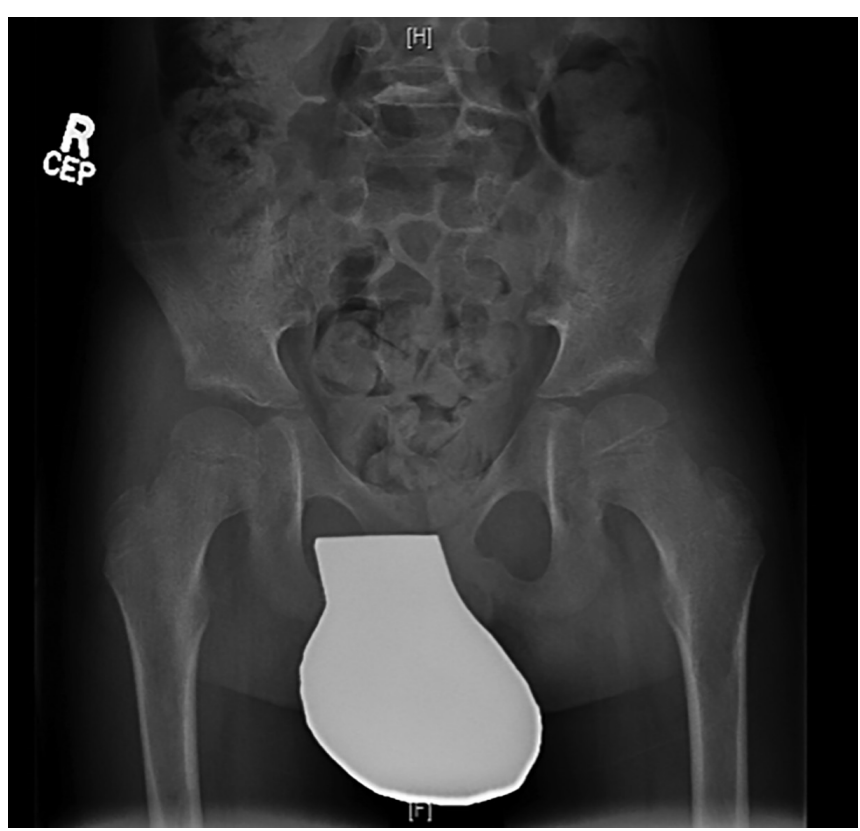

Figure 1: Anteroposterior radiograph of the hips of a six-year-old boy with autism spectrum disorder and left hip pain, showing bilateral osteopenia of the femoral heads, which is most marked in the right hip. 


\section{What is the most likely diagnosis?}

a. Leukemia

b. Henoch-Schönlein purpura

c. Bleeding disorder

d. Nonaccidental injury

e. Dietary deficiencies
The answer is (e). Given the patient's normal leukocyte and platelet counts, and the absence of blasts on peripheral smear, leukemia was not likely. In addition, although the hemoglobin concentration was low, the reticulocyte count was appropriately elevated, which indicated adequate bone marrow function. Henoch-Schönlein purpura can cause both joint pain and palpable purpura without thrombocytopenia; however, the joint pain is typically more acute

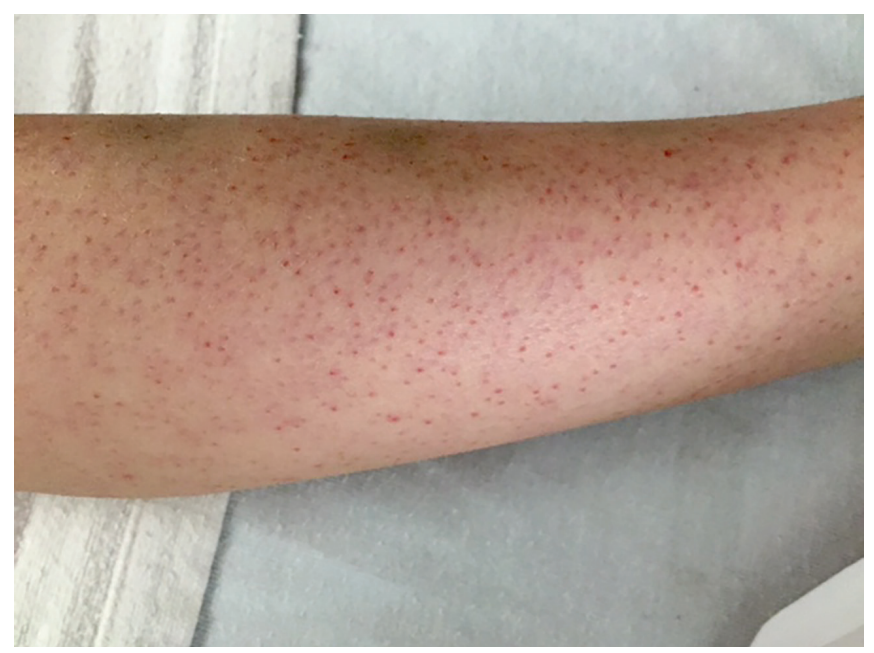

Figure 2: Palpable petechiae on the patient's arms and legs, shown here on his left leg.

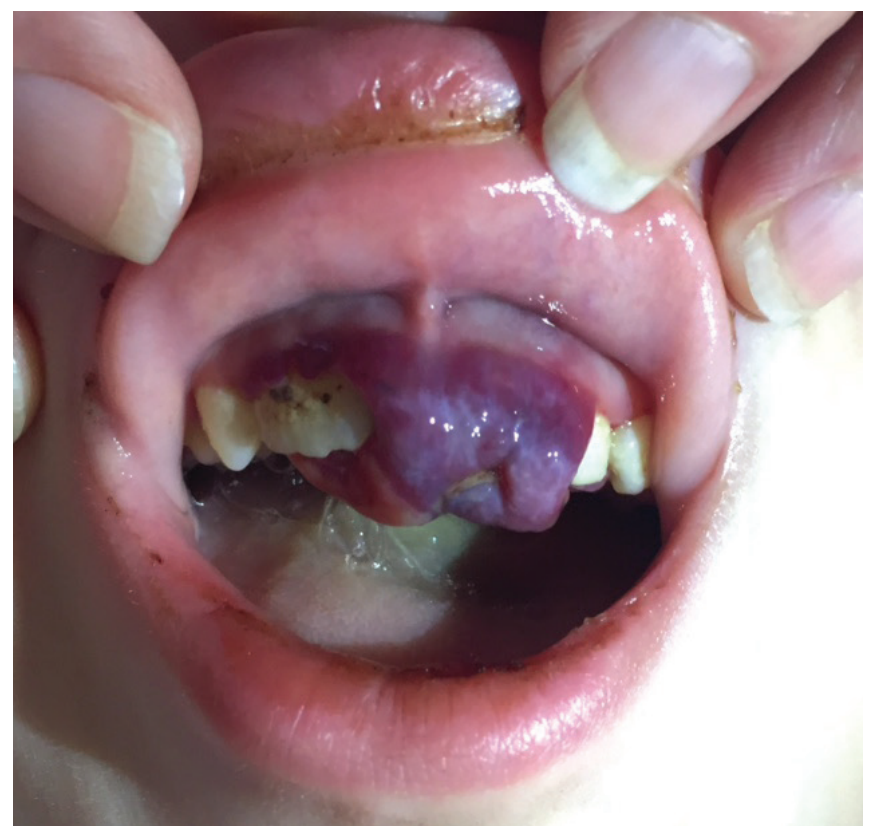

Figure 3: Swollen, purple gingiva with blood-filled blisters. in onset. In addition, mucosal bleeding and anemia are not characteristic. A bleeding disorder would be less likely in the context of a normal platelet count, and normal international normalized ratio and partial thromboplastin time. Nonaccidental injury should be considered in any case of bony pain and unexplained bleeding, but it was not felt to be the cause in view of the other findings.

Dietary deficiencies should be considered in children presenting with anemia, gingival disease and osteopenia, especially those with autism spectrum disorder, who are more likely to have restrictive diets. A 24-hour dietary recall interview was conducted. We established the percentage of recommended daily intake of vitamins and minerals by looking at the specific brands purchased.

Our patient had a highly restrictive diet, with complete absence of vitamins $C$ and $D$ and only minimal iron intake (Box 2). He had not consumed fruits (other than a few apples) or vegetables in more than three years and was not taking vitamin supplements. He ate the same foods every day, which meant the 24-hour dietary recall reflected his entire diet. At the time of our examination, we learned that a dietitian had started working with the patient to reintroduce fruits and vegetables. Apples were the first fruit reintroduced, several months earlier, but the patient had stopped eating them because of gingival pain.

The combination of bony pain, perifollicular petechial rash, bleeding gums, anemia and no dietary vitamin $C$ is classic of scurvy. To confirm the diagnosis, ascorbic acid measurement was done, as well as iron studies and measurement of vitamins $B_{12}$ and D (Box 3). Zinc levels were not measured at the time of diagnosis.

Although the patient's dietary history suggested micronutrient deficiency secondary to restriction, deficiency from malabsorption was also considered. This was thought to be less likely considering the degree of dietary restriction and the absence of gastrointestinal symptoms. The decision was therefore made to start vitamin and mineral supplementation ( $250 \mathrm{mg}$ of vitamin C and $800 \mathrm{IU}$ of vitamin D once daily, $40 \mathrm{mg}$ of elemental iron and $250 \mathrm{mg}$ of calcium carbonate three times daily, and a multivitamin once daily) and to monitor

\section{Box 1: Initial laboratory test results}

\begin{tabular}{|lcc|}
\hline Laboratory test & Value & $\begin{array}{c}\text { Reference } \\
\text { range* }\end{array}$ \\
\hline \begin{tabular}{l} 
Leukocyte count, $\times 10^{9} / \mathrm{L}$ \\
\hline Platelet count, $\times 10^{9} / \mathrm{L}$
\end{tabular} & 6.3 & $4.0-10.0$ \\
\hline Hemoglobin, $\mathrm{g} / \mathrm{L}$ & 250 & $150-400$ \\
\hline Mean corpuscular volume, $\mathrm{fL}$ & 74 & $120-160$ \\
\hline Reticulocyte count, $\times 10^{9} / \mathrm{L}$ & 79 & $77-96$ \\
\hline Blood smear & $153(5.36 \%)$ & $10-100$ \\
\hline Erythrocyte sedimentation & Moderate & \\
\hline rate, mm/h & polychromasia & $2-34$ \\
\hline C-reactive protein, $\mathrm{mg} / \mathrm{L}$ & 103 & $0.1-1.0$ \\
\hline International normalized ratio & & $0.80-1.20$ \\
\hline Partial thromboplastin time, s & 31.1 & $24.0-36.0$ \\
\hline *Reference ranges for age. & 1.0 & \\
\hline
\end{tabular}


the patient closely through follow-up appointments to determine the need for further investigations into malabsorption.

Within two weeks, the patient's hip pain had resolved and his gingival bleeding had greatly improved. Results of repeat laboratory investigations done eight weeks later showed normalization of the erythrocyte sedimentation rate, hemoglobin concentration and vitamin D level (Box 3). Because of laboratory error, the repeat ascorbic acid level is not reported here. The rapid resolution of symptoms and normalization of laboratory values following vitamin and mineral supplementation further indicated that the micronutrient deficiencies were secondary to dietary restriction rather than to malabsorption. No further investigations were pursued.

\section{Discussion}

Although scurvy is thought of as a disease of the past, it still occurs today. The incidence of clinical scurvy in North America is unknown, but the overall prevalence of vitamin $\mathrm{C}$ deficiency in individuals more than six years old in the United States is 3.6\%, based on data from the National Health and Nutrition Examination Survey. ${ }^{1}$

Vitamin C, or ascorbic acid, plays a vital role in the synthesis and cross-linking of collagen. ${ }^{2}$ Many of the clinical manifestations of scurvy can be attributed to capillary fragility, secondary to depletion of pericapillary collagen. ${ }^{3}$

The most common radiographic finding is osteopenia, ${ }^{3}$ which is nonspecific, and in our case may have been magnified by concomitant vitamin D deficiency, in addition to zinc deficiency. Less common but more specific are radiographic findings that were not seen in our patient, including calcification zones with adjacent lucency in the distal metaphyses, known as the "scurvy zone," which can be the site of fractures. ${ }^{3}$ Healing fractures in this zone can lead to spur formation, known as Pelkan spurs. ${ }^{3}$ In addition, the border of the epiphyses can be increased in density, known as a Wimberger ring. ${ }^{3}$ These findings are often unfamiliar to radiologists because of the rarity of scurvy. ${ }^{3}$

Restrictive diets are not uncommon in children with autism spectrum disorder. In a study comparing food selectivity in 111 children, Bandini and colleagues ${ }^{4}$ found that typically developing children refused $19 \%$ of foods offered, whereas those with autism spectrum disorder refused $42 \%$. Hyman and colleagues ${ }^{5}$ found that reported intakes of vitamin A, vitamin C and zinc were lower among children four to eight years of age with autism spectrum disorder than among matched controls. In addition, although it is often thought that children outgrow their picky eating habits, the proportion of children with nutrient intake less than the estimated average requirement increased with age for certain nutrients, including vitamins $A, C, E, B_{12}$ and folate, and the minerals zinc and magnesium. ${ }^{5}$

Scurvy has been reported in children with restrictive diets associated with varying degrees of developmental delay. ${ }^{3}$ It has also been reported in an infant fed exclusively almond beverages ${ }^{6}$ and in a child with end-stage renal disease whose loss of ascorbic acid

\section{Box 2: 24-hour dietary recall}

Vitamin/mineral; \% RDI

\begin{tabular}{llccc} 
Meal & \multicolumn{1}{c}{ Food } & Vitamin C & Vitamin D & Iron \\
Breakfast & $\begin{array}{l}\text { - Oatmeal raisin cookies } \\
\text { (2) (from fast-food } \\
\text { restaurant) } \\
\text { - Cola beverage }\end{array}$ & 0 & 0 & 6 \\
Snack & $\begin{array}{l}\text { Plain Greek yogurt } \\
\text { (3 spoonfuls) }\end{array}$ & 0 & 0 & 0 \\
Lunch & $\begin{array}{l}\text { - Chicken nuggets (2) } \\
\text { - Water }\end{array}$ & 0 & 0 & 4 \\
Snack & $\begin{array}{l}\text { Nacho cheese-flavoured } \\
\text { tortilla chips (small pack) }\end{array}$ & 0 & 0 & 0 \\
Dinner & $\begin{array}{l}\text { - Chicken nuggets (5) } \\
\text { - Cola beverage }\end{array}$ & 0 & 0 & 10 \\
\hline Snack & Microwave popcorn & 0 & 0 & 0 \\
\hline Total & $\quad 0$ & 0 & 20 \\
\hline Note: RDI = recommended daily intake. & & & \\
\hline
\end{tabular}

Box 3: Results of laboratory tests

\begin{tabular}{|c|c|c|c|}
\hline Laboratory test & Initial value & Value at 8-wk follow-up & Reference range* $^{*}$ \\
\hline Ascorbic acid, $\mu \mathrm{mol} / \mathrm{L}$ & $<5$ & NA & $>24$ \\
\hline Hemoglobin, g/L & 74 & 138 & $120-160$ \\
\hline Iron, $\mu \mathrm{mol} / \mathrm{L}$ & 3 & 16 & $5-27$ \\
\hline Ferritin, $\mu \mathrm{g} / \mathrm{L}$ & 110 & 140 & $14-80$ \\
\hline Transferrin, g/L & 2.19 & NA & $2.16-3.30$ \\
\hline Cyanocobalamin (vitamin $\mathrm{B}_{12}$ ), pmol/L & 360 & 425 & $220-1300$ \\
\hline Folate, $\mathrm{nmol} / \mathrm{L}$ & NA & 41 & $>30$ \\
\hline 25-hydroxyvitamin D, nmol/L & 17 & 109 & $70-250$ \\
\hline Alkaline phosphatase, U/L & 92 & 138 & $151-342$ \\
\hline Erythrocyte sedimentation rate, $\mathrm{mm} / \mathrm{h}$ & 103 & 30 & $2-34$ \\
\hline
\end{tabular}


was linked to peritoneal dialysis. ${ }^{7}$ In adults, scurvy has been reported in patients with restrictive diets and eating disorders, ${ }^{8}$ patients with either suspected or perceived ascorbic acid allergy ${ }^{9}$ and patients with diseases causing malabsorption, such as Whipple disease $^{10}$ and Crohn disease. ${ }^{8}$

Clinicians involved in the care of people with autism spectrum disorder should be prepared to counsel families regarding the risks of restrictive diets and the importance of vitamin supplementation when necessary. They should consider the possibility of insufficient dietary intake in these patients, look for signs and symptoms of nutritional deficiencies and refer to a dietitian for assessment if the patient appears to have a restrictive diet or signs and symptoms associated with nutritional deficiency.

\section{References}

1. NHANES 2005-2006. Atlanta: US Centers for Disease Control and Prevention. Available: www.cdc.gov/nchs/nhanes/search/nhanes05_06.aspx (accessed 2016 Oct. 19).

2. Amos LE, Carpenter SL, Hoeltzel MF. Lost at sea in search of a diagnosis: a case of unexplained bleeding. Pediatr Blood Cancer 2016;63:1305-6.

3. Weinstein M, Babyn P, Zlotkin S. An orange a day keeps the doctor away: scurvy in the year 2000. Pediatrics 2001;108:E55.

4. Bandini LG, Anderson SE, Curtin C, et al. Food selectivity in children with autism spectrum disorders and typically developing children. J Pediatr 2010;157:259-64.
5. Hyman SL, Stewart PA, Schmidt B, et al. Nutrient intake from food in children with autism. Pediatrics 2012;130(Suppl 2):S145-53.

6. Vitoria I, López B, Gómez J, et al. Improper use of a plant-based vitamin C-deficient beverage causes scurvy in an infant. Pediatrics 2016;137:e20152781.

7. Kittisakmontri K, Swangtrakul N, Padungmaneesub W, et al. Gingival bleeding and bloody dialysate: a case report of scurvy in a child with end-stage renal disease receiving peritoneal dialysis. J Ren Nutr 2016;26:407-11.

8. Levavasseur M, Becquart C, Pape E, et al. Severe scurvy: an underestimated disease. Eur J Clin Nutr 2015;69:1076-7.

9. Smith A, Di Primio G, Humphrey-Murto S. Scurvy in the developed world. CMAJ 2011;183:E752-5.

10. Hmamouchi I, Costes V, Combe B, et al. Scurvy as the presenting illness of Whipple's disease exacerbated by treatment with etanercept in a patient with ankylosing spondylitis. J Rheumatol 2010;37:1077-8.

Competing interests: None declared.

This article has been peer reviewed.

The authors have obtained patient consent.

Affiliation: Division of Paediatric Medicine, Department of Paediatrics, The Hospital for Sick Children, Toronto, Ont.

Contributors: Both authors contributed equally to the work, drafted and critically revised the manuscript, approved the final version to be published and agreed to act as guarantors of the work.

Correspondence to: Melanie Conway, melanie.conway@sickkids.ca 